\title{
Credit Score Analysis for Loan using Raw Data Through Third Party Application
}

\author{
Shivam Gaur, Rajani Katiyar, Umashankar S.
}

\begin{abstract}
Credit Score Analysis for Loan using raw data through third party Application has the potential to meet the needs for high speed Credit Information Bureau India Limited (CIBIL) score calculation. The aim is to increase fairness and reduce human intervention in this calculation process by passing all the data through software process and following the unbiased flow. The need to increase fairness among the disbursement of Loans has pushed this process to its limits. In this paper the required process flow to obtain CIBIL score is discussed.

Keywords : CIBIL, No Structured Query Language (NoSQL), Structured Query Language (SQL), Spring Framework, IntelliJ, JavaScript Object Notation (JSON), Application Program Interface (API).
\end{abstract}

\section{INTRODUCTION}

CIBIL score analysis is a very long and tedious process with a lot of manual intervention in analysis of data points through obtained data. Credit Score Analysis for Loan using raw data through third party Application will provide a more optimal flow for this process with less constraint, more reliable method and reducing the manpower required. Process flow aims to take raw customer data and pass it to the third party application which will help to verify important information about the customer. The process will act as a support program that will help the organizations to obtain the data of customers and provide validations on the report.

\section{DATA FLOW DESIGN}

The initial data that is obtained from customer is in huge quantity and not having all the fields filled as well. So, this data should be stored in NoSQL database, as in this database the query execution and processing speed will be faster as compared to SQL database. The major factor for this will be storing the data in JSON form and not in table as there is no relational data formed. After the initial data is obtained the

Revised Manuscript Received on May 07, 2020.

* Correspondence Author

Shivam Gaur*, Electronics and Communication Engineering Department, R.V. College of Engineering, Bengaluru, India. Email: shivamgaur.ec16@rvce.edu.in

Rajani Katiyar, Electronics and Communication Engineering Department, R.V. College of Engineering, Bengaluru, India. Email: rajanikatiyar@rvce.edu.in

Umashankar S, Software Developer Engineer, Lendingkart Technologies Pvt. Ltd, Bengaluru, India. Email: umashankar.s@lendingkart.com

(C) The Authors. Published by Blue Eyes Intelligence Engineering and Sciences Publication (BEIESP). This is an open access article under the CC BY-NC-ND license (http://creativecommons.org/licenses/by-nc-nd/4.0/) organization can focus to obtain all the required information about the genuinely interested customers. This can happen through different contact and communication channels. Once all the required information is obtained and the customer is verified the data can be moved to the SQL database as now the data has passed through the funnel and filtered customers so the data is also not as huge as initial data and has all the required fields filled. Database which is open-source and relational should be used. MySQL is one such relational database management system (RDBMS). Further Processing will happen with data in MySQL database only. Fig. 1 depicts the design flow followed in the process.

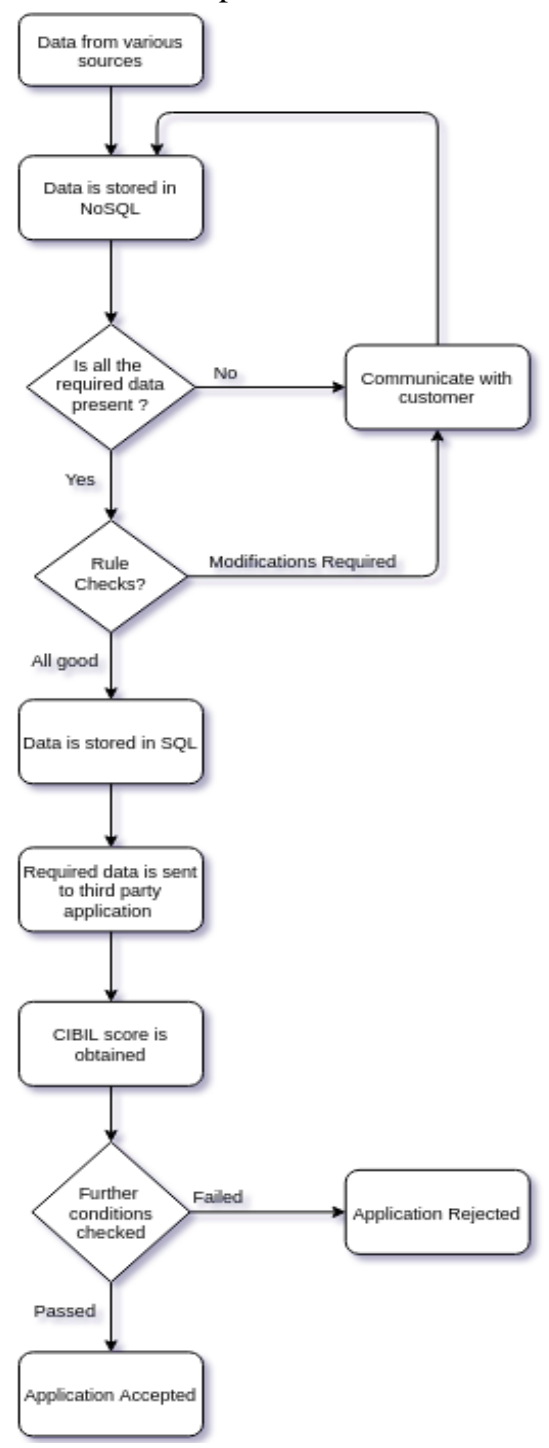

Fig. 1. Design Flow

Published By:

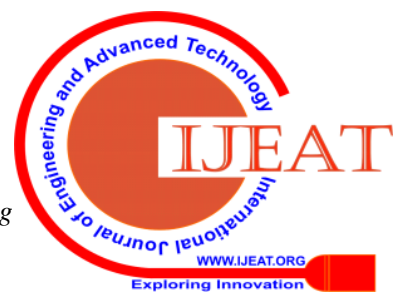




\section{METHODOLOGY ADOPTED}

Initial customer data can be obtained done through different channels such as website, mobile app, social media platforms, etc. After the details are obtained by the organization the various rule check can be applied like if the Permanent Account Number (PAN) details are there. These rule checks are done at the backend in Java.

Java is used as it is portable, meaning it is platform independent and the programs run similarly on all platforms. This is made possible through JVM which compiles Java byte code to machine code for each platform. Java logo is shown in Fig. 2.

The framework used is spring framework, the logo for which is shown in Fig. 3, as the spring framework provides infrastructural support at the application level and provides configuration for the program to be deployed on any kind of environment.

The suitable integrated development environment (IDE) that is used is IntelliJ. IntelliJ itself is written in Java and is used for developing software applications. Code completion, code refactoring, code debugging are some unique features of IntelliJ.

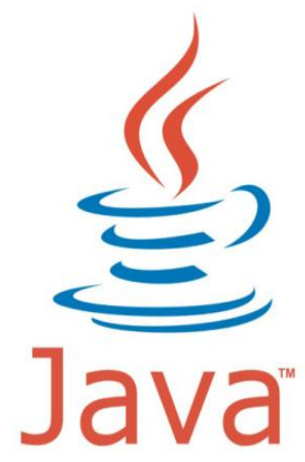

Fig. 2. Java Logo

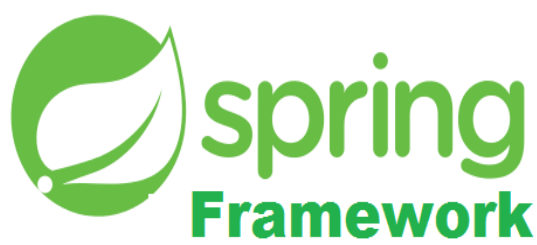

Fig. 3. Spring Framework Logo

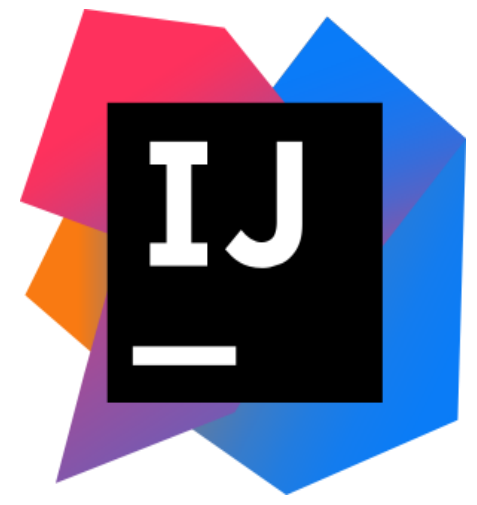

Fig. 4. IntelliJ Logo
The rule checks can vary from the income of the customer to income tax return filing depends on what basis the organizations starts to trust its customers.

Once the rule checks are complete the application of the customer can be forwarded to the third party application for CIBIL score calculation but only with the required details as the customer data needs to be kept confidential.

The third party application will provide the CIBIL score of the customer. Once that is obtained the organization can do further checks to decide whether to give Loan to the customer or not. If the checks are not successful and the customer application fails at any point than loan allotment will be rejected if the checks are successful and application succeeds through all the checkpoints than the loan can be allotted to the customer.

\section{RESULTS AND DISCUSSIONS}

For the process flow mentioned the performance improvement was observed as queries execution and completion took less time. The data segregation between structured and unstructured databases has increased processing speed saving essential time limits, API's developed through REST are working flawlessly, also it was observed that moving ahead of the orthodox methods has increased productivity and CIBIL score calculation now involves less human intervention.

\section{COCLUSION}

From the obtained results and what is discussed above it is clear that the process has provided success in achieving the required goal of reducing human intervention and reducing time required in calculating CIBIL score to grant loans. This method provides a hope for future to implement it in more fruitful manner.

\section{REFERENCES}

1. Kavya Guntupally, Ranjeet Devarakonda, Kenneth Kehoe "Spring Boo based REST API to Improve Data Quality Report Generation for Big Scientific Data: ARM Data Center Example", IEEE International Conference on Big Data (Big Data) - Seattle, WA, USA- 10-13 Dec. 2018.

2. Gabriel Menezes, Bruno Cafeo, Andre Hora "Framework Code Samples: How Are They Maintained and Used by Developers?", ACM/IEEE International Symposium on Empirical Software Engineering and Measurement (ESEM)- Porto de Galinhas, Recife, Brazil- 19-20 Sept. 2019.

3. Yunhua Gu, Shu Shen, Jin Wang, Jeong-Uk Kim "Application of NoSQL Database MongoDB ", International Conference on Consumer Electronics(ICCE)- Taipei, Taiwan- 6-8 June 2015.

4. Julio César Cortés Ríos, Kamilla Kopec-Harding, Sukru Eraslan, Christopher Page, Robert Haines, Caroline Jay and Suzanne M. Embury "A Methodology for Using GitLab for Software Engineering Learning Analytics", EEE/ACM 12th International Workshop on Cooperative and Human Aspects of Software Engineering- Montreal, Canada- 27 May 2019.

5. Kodrat Iman Satoto, R. Rizal Isnanto, Rinta Kridalukmana, Kurniawan Teguh Martono "Optimizing MySQL database system on information systems research, publications and community service", 2016 3rd International Conference on Information Technology, Computer, and Electrical Engineering (ICITACEE), Semarang, Indonesia- 19-20 Oct. 2016.

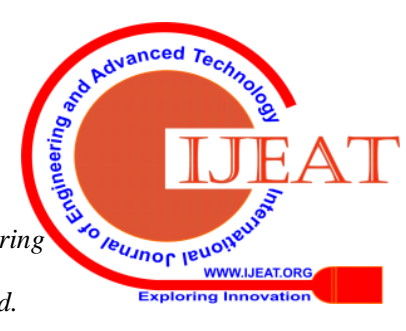


6. A. Bazghandi "Web Database Connectivity Methods (using Mysql) in Windows Platform", 2006 2nd International Conference on Information \& Communication Technologies, Damascus, Syria- 24-28 April 2006.

7. Aditya Singh, Piyush Chawla, Karan Singh, Ashutosh Kumar Singh "Formulating an MVC Framework for Web Development in JAVA", 2018 2nd International Conference on Trends in Electronics and Informatics (ICOEI), Tirunelveli, India- 11-12 May 2018.

8. Zeljko Jovanovic, Dijana Jagodic, Dejan Vujicic, Siniša Randić "Java Spring Boot Rest WEB Service Integration with Java Artificial Intelligence Weka Framework", UNITEH 2017, International Scientific Conference, Gabrovo, Bulgaria- November 2017.

\section{AUTHORS PROFILE}

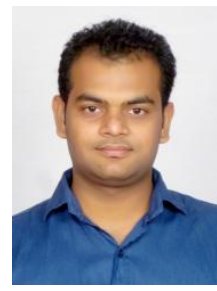

Shivam Gaur is currently pursuing his Bachelor of Engineering Degree as a student of 8th Semester from Electronics and Communication Department, R.V. College of Engineering, Bengaluru. $\mathrm{He}$ is also working as an Intern Software Developer Engineer with Lendingkart Pvt. Ltd, Bengaluru since January 2020. His 1 paper is under publication with a National Conference. He has worked on some of the technologies and frameworks listed - Spring Boot, Java, Hibernate, MongoDB, MySQL. His areas of interest include Software Development and Web Security.

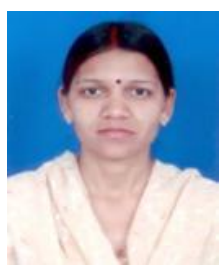

Rajani Katiyar is working as Assistant Professor, Department of Electronics and Communication Engineering, RV college of Engineering, Bengaluru. She has got 14 years of teaching experience. She has obtained Bachelor of technology degree from MJPRU Bareilly in the year 2001 and obtained Master of Technology degree from DAVV University, Indore in 2005. She completed her PhD degree in the year 2020 from Visveswaraya Technological University, Belagavi, India. She has published 1 paper in National conferences, 3 papers in IEEE International conferences and 5 papers in International journals. Her area of interests includes Broadband Communication and Signal Processing.

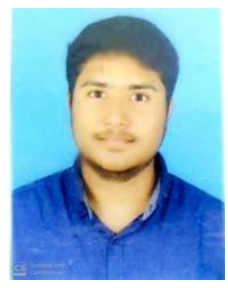

Umashankar $\mathbf{S}$ is working as a Software Developer Engineer - 1 in Lendingkart Technologies Pvt. Ltd, Bengaluru. He has got 1 year and 1 month of experience as a software developer. He has obtained Bachelor of Engineering degree from PES Institute of Technology [West Campus, Bengaluru] in the year 2018. He has worked on some of the technology and framework listed - Spring Boot, Spring Mvc, Java, Elastic Search, Hibernate, Mongo, Kafka etc. 